\title{
Shear Viscosity from Lattice QCD
}

\author{
Szabolcs Borsányi, ${ }^{a}$ Zoltán Fodor, ${ }^{a b c}$ Simon Mages, ${ }^{*}{ }^{d e f}$ Andreas Schäfer, ${ }^{d}$ and \\ Kálmán K. Szabóabd \\ ${ }^{a}$ University of Wuppertal, Department of Physics, Wuppertal D-42097, Germany \\ ${ }^{b}$ Jülich Supercomputing Center, Jülich D-52425, Germany \\ ${ }^{c}$ Eötvös University, Budapest 1117, Hungary \\ ${ }^{d}$ University of Regensburg, Department of Physics, Regensburg D-93053, Germany \\ ${ }^{e}$ Utrecht University, Institute for Theoretical Physics, 3584 CE Utrecht, The Netherlands \\ ${ }^{f}$ CERN, Theory Group, CH-1211, Geneva 23, Switzerland \\ E-mail: borsanyi@uni-wuppertal.de, fodorabodri.elte.hu, \\ simon-wolfgang.mages@ur.de, andreas.schaeferdur.de, \\ szaboka@general.elte.hu
}

Understanding of the transport properties of the the quark-gluon plasma is becoming increasingly important to describe current measurements at heavy ion collisions. This work reports on recent efforts to determine the shear viscosity $\eta$ in the deconfined phase from lattice QCD. The main focus is on the integration of the Wilson flow in the analysis to get a better handle on the infrared behaviour of the spectral function which is relevant for transport. It is carried out at finite Wilson flow time, which eliminates the dependence on the lattice spacing. Eventually, a new continuum limit has to be carried out which sends the new regulator introduced by finite flow time to zero. Also the non-perturbative renormalization strategy applied for the energy momentum tensor is discussed. At the end some quenched results for temperatures up to $4.5 T_{c}$ are presented.

The 32nd International Symposium on Lattice Field Theory

23-28 June, 2014

Columbia University New York, NY

${ }^{*}$ Speaker. 


\section{Introduction}

\subsection{Motivation and Outline}

The main aim of present day high-energy heavy ion physics is to determine medium properties of the quark gluon plasma or more precisely the size of its transport coefficents [1]. In this context the ratio $\eta / s$, i.e. shear viscosity over entropy density, is of special interest as there are strong arguments for the existence of a lower bound of $1 / 4 \pi$ for gauge theories in the strong coupling limit. This was in particular worked out within AdS/CFT duality [2].

Since decades lattice QCD studies have been published on that topic [3, 4, 5, 6, 7, 8]. However, some of them suffer from inherent problems in accessing the low energy behaviour of the shear spectral function due to some properties of the finite temperature integral kernel $K(\omega, \tau)$ which links the spectral functions to Euclidean correlators, the quantities which are accessible by lattice QCD calculations. These properties make it quite difficult to extract the low energy behaviour of spectral functions.

In the last years smearing was used very successfully to reduce the influence of high energy properties of lattice configurations and to reveal low energy properties [9]. In particular, the Wilson flow proved to be a valuable tool for lattice QCD, e.g. for scale setting and determination of the anisotropy [10,11], and for the renormalization of the energy momentum tensor [12, 13]. We try to employ these methods to increase the sensitivity of the analysis to the low energy part of the spectral functions.

Using smearing as a noise reduction technique instead of the multi-level algorithm in $[7,8]$ is crucial for dynamical simulations, as in that case multi-level algorithms are not available. This is because the action in full QCD is non-local after integrating out the fermionic degrees of freedom. So an alternative strategy is required for results with dynamical fermions.

\subsection{Transport from Spectral Functions}

Information about the viscosity is encoded in correlators of the traceless part $\Theta_{\mu \nu}$ of the energy momentum tensor $T_{\mu \nu}$. For pure gauge theories it is defined as

$$
\Theta_{\mu v}(x)=F_{\mu \sigma}(x) F_{v \sigma}(x)-\frac{1}{4} \delta_{\mu v} F_{\rho \sigma}(x) F_{\rho \sigma}(x),
$$

where $F_{\mu v}(x)$ is the field-strength tensor at $x=(\tau, \mathbf{x})$ and a trace over the suppressed color indices is understood.

The Euclidean correlators, which can be measured on the lattice, are defined as

$$
C_{\mu v, \rho \sigma}(\tau, \mathbf{q})=T^{-5} \int d^{3} \mathbf{x} e^{i \mathbf{q} \cdot \mathbf{x}}\left(\left\langle\Theta_{\mu v}(x) \Theta_{\rho \sigma}(0)\right\rangle-\left\langle\Theta_{\mu v}(x)\right\rangle\left\langle\Theta_{\rho \sigma}(0)\right\rangle\right) .
$$

These correlators are connected to their spectral functions $\rho_{\mu v, \rho \sigma}(\tau, \mathbf{q})$ via an integral transformation

$$
\begin{aligned}
C_{\mu v, \rho \sigma}(\tau, \mathbf{q}) & =\int_{0}^{\infty} \mathrm{d} \omega K(\tau, \omega) \rho_{\mu v, \rho \sigma}(\omega, \mathbf{q}) \\
K(\tau, \omega) & =\frac{\cosh (\omega(\tau-1 /(2 T)))}{\sinh (\omega /(2 T))}
\end{aligned}
$$


with the finite temperature kernel $K(\tau, \omega)$. Transport properties then follow from the low energy limit of the spectral functions via Kubo formulae. For the two channels which are relevant for the viscosity these are $[14,8]$

$$
\pi \lim _{\omega \rightarrow 0} \lim _{q \rightarrow 0} \frac{\rho_{13,13}(\omega, q)}{\omega}=\eta, \quad \pi \lim _{\omega \rightarrow 0} \lim _{q \rightarrow 0} \frac{\rho_{33,33}(\omega, q)}{\omega}=\frac{4}{3} \eta+\zeta
$$

with the bulk viscosity $\zeta$ and the momentum taken parallel to the 3-direction $\mathbf{q}=q \mathbf{e}_{3}$. The bulk viscosity is at the temperatures relevant for phenomenology generally much smaller than the shear viscosity $\eta$. Therefore also $\rho_{33,33}$ can be used to determine the shear viscosity $\eta$.

\subsection{Lattice Setup}

So far we have performed only a preparatory study for future dynamical simulations. We use pure $\mathrm{SU}(3)$ gauge theory, more precisely, a Symanzik improved gauge action with $O\left(a^{2}\right)$ discretization errors $[15,16]$. One should keep in mind, that pure $S U(3)$ theory is physically very different from full QCD, e.g. it has a first order phase transition instead of a crossover [17]. The traceless energy momentum tensor $\Theta_{\mu \nu}$ is built directly from the clover lattice field strength tensor $F_{\mu \nu}$ and has $O\left(a^{2}\right)$ discretization errors. In this effort configurations at temperatures ranging from $0.75 T_{c}$ to $4.0 T_{c}$ were generated for $8 \leq n_{t} \leq 16$ and aspect ratios from 1 to 8 . An update sweep consists of one heatbath and four overrelaxation steps.

The simulations were performed on the QPACE machine [18] on the Wuppertal site.

\subsection{Smearing and the Wilson Flow}

Stout Smearing The smearing of configurations before measurement of observables is a widely used technique in lattice QCD since a long time. The smearing dampens ultraviolet fluctuations and has a large impact on the statistical error of observables. Keeping the smearing fixed in lattice units while performing a continuum limit recovers the full theory including the ultraviolet part. A widely used smearing procedure is stout smearing [19], which we also use in this study.We define a stout smearing radius in dependence of the stout smearing parameter $\rho_{\text {Stout }}$ and the number of stout smearing iterations $N_{\text {Stout }}$ as

$$
r_{\text {smear }}=a \sqrt{8 \rho_{\text {Stout }} N_{\text {Stout }}} .
$$

Wilson Flow A particularly interesting and promising concept in lattice QCD is the Wilson flow [9]. It can be used as a tool for understanding topological properties of the gauge fields, as an alternative method to set the scale in lattice calculations, and as an alternative method to determine the gauge anisotropy $[11,10]$.

This flow is generated by infinitesimal stout smearing steps and is therefore a smoothing operation on gauge configurations. This is directly seen by its action on the gauge fields in leading order of perturbation theory in the bare coupling $g_{0}$ [9]

$$
A_{\mu}^{t}(x)=g_{0} \int d^{4} y K_{t}(x-y) A_{\mu}(y)+O\left(g_{0}^{2}\right), K_{t}(z)=\frac{e^{-z^{2} / 4 t}}{(4 \pi t)^{2}} .
$$

This makes the smoothing effect explicit with a radius of $\sqrt{8 t}=r_{\text {smear }}$.

It has also been shown, that all observables measured at finite Wilson flow time are finite renormalized quantities and in particular with removed discretization errors [9]. 


\section{Energy Momentum Tensor Renormalization}

The traceless energy momentum tensor renormalizes multiplicatively with different renormalization factors for diagonal and off-diagonal entries. Here we only consider diagonal entries of this tensor and determine this single factor $Z^{\text {diag }}$ by matching our lattice quantities to results from thermodynamics [20]. In particular we use the value for the renormalized entropy density $s=(\varepsilon+p) / T$ and calculate the renormalization factor via

$$
\frac{s T}{4}=\left\langle\Theta_{11}\right\rangle=Z^{\text {diag }}\left\langle\theta_{11}\right\rangle
$$

by measuring the unrenormalized value $\left\langle\theta_{11}\right\rangle$ on the lattice.

To access renormalized correlators of off-diagonal components we use the general tensor decomposition of correlators of the energy momentum tensor as given in [3, 7]. For the shear channel one gets

$$
\left\langle\Theta_{12}(\tau, \mathbf{x}) \Theta_{12}(0, \mathbf{0})\right\rangle=\frac{1}{4}\left\langle\left(\Theta_{11}-\Theta_{22}\right)(\tau, \mathbf{x})\left(\Theta_{11}-\Theta_{22}\right)(0, \mathbf{0})\right\rangle .
$$

\section{Analysis Strategy}

\subsection{Choice of the Ansatz for the Spectral Function}

In this work we use finite momentum data which has to be reflected in the ansatz. For the low energy range the predictions from hydrodynamics [14] can be used as long as $\omega, q<\pi T$. For the relevant channels these predictions imply

$$
\begin{aligned}
& \frac{\rho_{13,13}(\omega, q)}{\omega} \stackrel{\omega, q \rightarrow 0}{\longrightarrow} \frac{\eta}{\pi} \frac{\omega^{2}}{\omega^{2}+\left(\eta q^{2} /(\varepsilon+p)\right)^{2}} \\
& \frac{\rho_{33,33}(\omega, q)}{\omega} \stackrel{\omega, q \rightarrow 0}{\longrightarrow} \frac{\Gamma_{s}}{\pi} \frac{(\varepsilon+p) \omega^{4}}{\left(\omega^{2}-v_{s}(q)^{2} q^{2}\right)^{2}+\left(\Gamma_{s} \omega q^{2}\right)^{2}}
\end{aligned}
$$

with the sound attenuation length $\Gamma_{s}=\left(\frac{4}{3} \eta+\zeta\right) /(\varepsilon+p)$ and the speed of sound $v_{s}(q)$. To fit the sound channel spectral function $\rho_{33,33}$ we use the same ansatz based on hydrodynamics, like in [8] apart from dropping the mid frequency contribution.

\subsection{Usage of the Wilson Flow}

Throughout this work the Wilson flow is approximated by stout smearing steps. However, in contrast to the standard usage of the smearing, we keep the smearing fixed in physical units and model the effect of this on the high frequency contributions directly in the spectral function. For this we include a weighting function in the spectral representation

$$
C_{33,33 ; \Omega}(\tau, q)=\int_{0}^{\infty} \mathrm{d} \omega K(\tau, \omega) W_{\Omega}(\omega) \rho_{33,33}(\omega, q) .
$$

The function $W_{\Omega}(\omega)$ depends on the smearing scale $\Omega=\pi / r_{\text {smear }}=\pi / \sqrt{8 t}$. We choose different parametrizations of $W_{\Omega}(\omega)$ and account for the variations introduced by that in the systematic error. Infrared quantities like transport coefficients are in general not sensitive to details of the 
parametrization, as it modifies only physics at higher energies than $\Omega$. Apart from decreasing the statistical errors, the smearing suppresses a large part of the high frequency background to the infrared physics in the spectral function, removes cutoff effects as long as $r_{\text {smear }}>a$, and also increases the number of data points, if one includes different cutoffs in a single global fit.

A remark on the behaviour of the correlator at short Euclidean times for finite flow times is in order. Following Eq. (1.7) the smearing evolves local operators to non-local ones with approximately Gaussian support in position space for large energies or weak coupling. Considering then the Euclidean correlator over such smeared operators yields a correlator which is also smeared in the Euclidean time distance because the Gaussians of the operators factorize. This leads to a negative curvature of the correlator for Euclidean time separations which are smaller than the smearing radius. This feature does not arise for the infrared behaviour at length scales much larger than the smearing radius, which is the relevant one for studying the transport properties of the theory. In the fits to measure $\eta / s$, we introduce a parameter $\tau_{\min }$ for the minimal Euclidean time used in the fit. It is always chosen larger than $r_{\text {smear }}$ such that only those parts of the correlator are used, where the curvature is positive. The exact choice of this parameter has been varied and the resulting change in $\eta / s$ is incorporated in the total systematic error.

\section{Lattice Results}

\subsection{Discretization Errors and Scaling}

In order to demonstrate the effect of removal of finite lattice spacing effects, a series of simulations at different lattice spacing is performed. Fig. 1 gives as an example the dependence of a shear channel correlator on lattice spacing for some selected values of the flow time. The plots for different correlators and for different temperatures exhibit the same features. For small smearing radii the discretization errors are sizable and the statistical errors are large for large separations in Euclidean time. For larger smearing radii the values for all the different lattice spacings lie essentially on the same curve and the discretization errors are negligible. The statistical error has been greatly reduced. The magnitude of the correlator at small Euclidean distances decreased by orders of magnitude with increasing smearing, just as expected from an increasing loss of the high energy parts of the spectrum in the spectral function.

Let us note here, that at a finite smearing radius no renormalization is needed. In order to extract physical information, first one has to normalize the correlators by a procedure completely analogous to the one in Sec. 2 and then do a limit of sending the smearing radius to zero. We have not considered this limit procedure in this work, instead to increase the signal we performed a simultaneous fit to a set of smearing radii in the range $1 / 4 T \lesssim r_{\text {smear }}<1 / 2 T$. A modification in finite distance properties should not have a large influence on results for infrared quantities. But it remains as an important task for the future to perform a controlled limit of the analysis to vanishing smearing radius.

\subsection{Determination of the Viscosity}

To access a larger number of small spatial momenta in the region of validity of hydrodynamics, $|\mathbf{q}| \leq \pi T$, two different spatial lattice sizes are used with aspect ratios $r_{a}=n_{s} / n_{t}$ of 6 and 8 . It was 

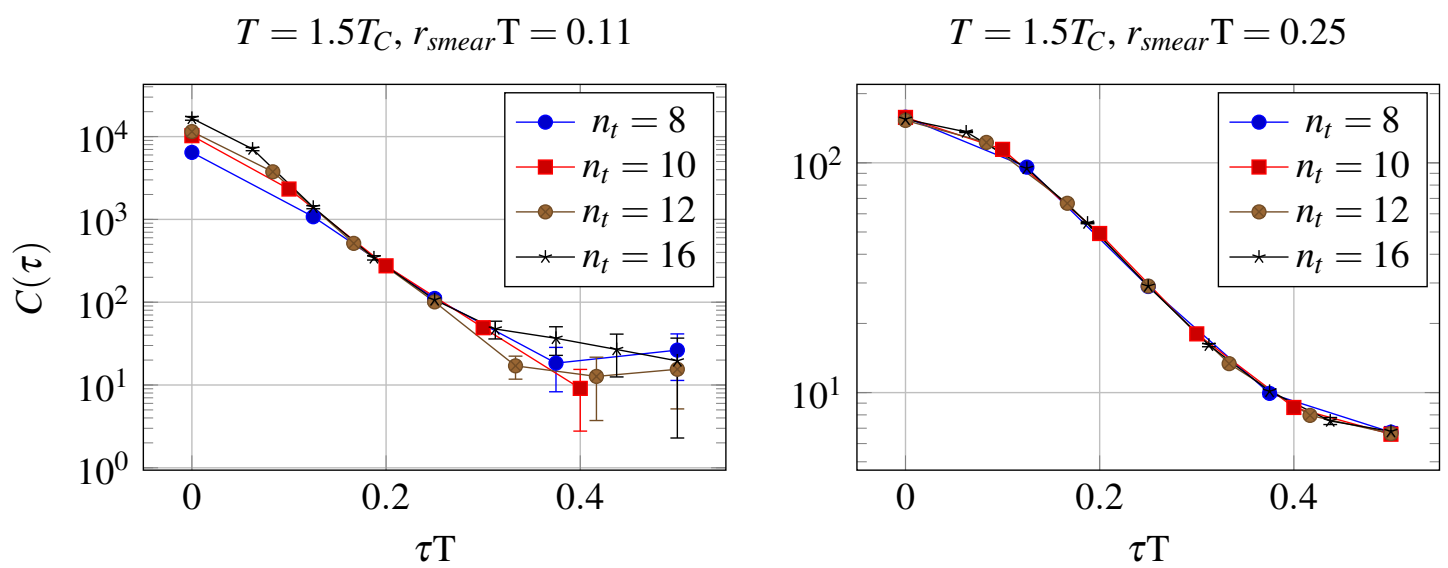

Figure 1: $C(\tau)=\frac{1}{T^{5}} \int \mathrm{d} \mathbf{x}\left\langle\Theta_{12}(0,0) \Theta_{12}(\tau, \mathbf{x})\right\rangle$ scaling behaviour. Errors are statistical jackknife errors.

checked that finite volume errors are negligible on these lattices. We use the two smallest non-zero momenta for both lattice sizes. For the determination of the viscosity we use $n_{t}=8$ lattices with between $9.4 \cdot 10^{3}$ and 5.6 $10^{4}$ configurations separated by 32 update sweeps.

The global fit was repeated for different choices of parameters, e.g. the minimum Euclidean time included in the fit, different initial conditions for the fit parameters, functional forms of the weight function $W$, and different included smearings in the fit. In total that amounts to 162 different analyses from which the systematic error of the result is calculated. The statistical error is calculated with the jackknife method.

\begin{tabular}{c|ccc}
$T / T_{c}$ & 1.5 & 3.0 & 4.5 \\
\hline$\chi^{2} / \mathrm{DOF}$ & $1.2(2)(6)$ & $2.2(3)(5)$ & $2.1(3)(8)$ \\
$\eta / s$ & $0.24(7)(6)$ & $0.32(5)(5)$ & $0.43(9)(7)$
\end{tabular}

Table 1: Results of the fits to a form of the spectral function suggested by hydrodynamics. DOF is the number of degrees of freedom. The first error is statistical, the second systematical.

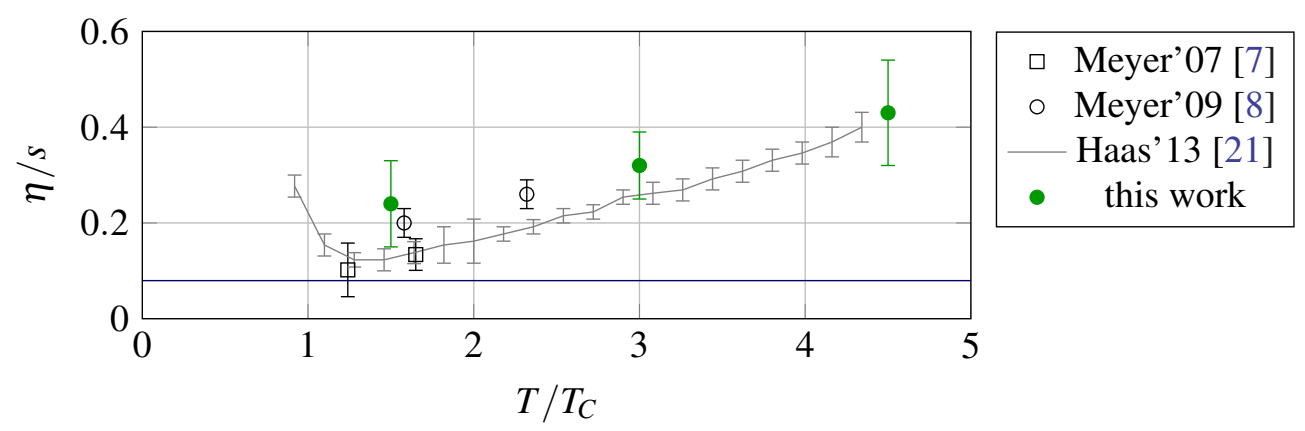

Figure 2: The results of the determination of the viscosity from this work compared to previously reported values in the literature. [21] is based on a functional renormalization group technique and no direct lattice study.

The preliminary fit results are listed in Tab. 1 . The values for $\eta / s$ have quite large statistical and systematical errors. As can be seen in Fig. 2 this makes a reliable statement on the dependence 
of the viscosity on temperature impossible. Our result for the viscosity is at the current error level consistent both with a constant and with the temperature dependence given in [21]. The figure also give the results of previous studies. This work is compatible with these results within the error. Even though our result has larger errors than the ones based on the multi-level algorithm [7,8], it shows that a determination of $\eta / s$ is in principle feasible without the multi-level algorithm using smearing techniques, i.e. in full QCD.

\section{Discussion and Outlook}

In this study we used the Wilson flow to increase the sensitivity of lattice studies to the infrared part of spectral functions as an alternative to multi-level algorithms in full QCD simulations. As test case this analysis determined the value of $\eta / s$ for different temperatures above $T_{c}$ from quenched lattice QCD.

\section{References}

[1] B. Müller, arXiv:1309.7616 [nucl-th].

[2] P. Kovtun, D. T. Son and A. O. Starinets, Phys. Rev. Lett. 94 (2005) 111601 [hep-th/0405231].

[3] F. Karsch and H. W. Wyld, Phys. Rev. D 35 (1987) 2518.

[4] S. Sakai, A. Nakamura and T. Saito, Nucl. Phys. A 638 (1998) 535 [hep-lat/9810031].

[5] A. Nakamura and S. Sakai, Phys. Rev. Lett. 94 (2005) 072305 [hep-lat/0406009].

[6] S. Sakai and A. Nakamura, PoS LAT 2005 (2006) 186 [hep-lat/0510100].

[7] H. B. Meyer, Phys. Rev. D 76 (2007) 101701 [arXiv:0704.1801 [hep-lat]].

[8] H. B. Meyer, Nucl. Phys. A 830 (2009) 641C [arXiv:0907.4095 [hep-lat]].

[9] M. Luscher, JHEP 1008 (2010) 071 [arXiv:1006.4518 [hep-lat]].

[10] S. Borsanyi, S. Durr, Z. Fodor, C. Hoelbling, S. D. Katz, S. Krieg, T. Kurth and L. Lellouch et al., JHEP 1209 (2012) 010 [arXiv:1203.4469 [hep-lat]].

[11] S. Borsanyi, S. Durr, Z. Fodor, S. D. Katz, S. Krieg, T. Kurth, S. Mages and A. Schäfer et al., arXiv:1205.0781 [hep-lat].

[12] L. Del Debbio, A. Patella and A. Rago, JHEP 1311 (2013) 212 [arXiv:1306.1173 [hep-th]].

[13] H. Suzuki, PTEP 2013 (2013) 8, 083B03 [arXiv:1304.0533 [hep-lat]].

[14] D. Teaney, Phys. Rev. D 74 (2006) 045025 [hep-ph/0602044].

[15] K. Symanzik, Nucl. Phys. B 226 (1983) 187.

[16] M. Lüscher and P. Weisz, Commun. Math. Phys. 97, 59 (1985) [Erratum-ibid. 98, 433 (1985)].

[17] Y. Aoki, G. Endrodi, Z. Fodor, S. D. Katz and K. K. Szabo, Nature 443 (2006) 675 [hep-lat/0611014].

[18] H. Baier, H. Boettiger, M. Drochner, N. Eicker, U. Fischer, Z. Fodor, A. Frommer and C. Gomez et al., PoS LAT 2009 (2009) 001 [arXiv:0911.2174 [hep-lat]].

[19] C. Morningstar and M. J. Peardon, Phys. Rev. D 69 (2004) 054501 [hep-lat/0311018].

[20] S. Borsanyi, G. Endrodi, Z. Fodor, S. D. Katz and K. K. Szabo, JHEP 1207 (2012) 056 [arXiv:1204.6184 [hep-lat]].

[21] M. Haas, L. Fister and J. M. Pawlowski, arXiv:1308.4960 [hep-ph]. 